\title{
Study on electrical properties of Ni-doped $\mathrm{SrTiO}_{3}$ ceramics using impedance spectroscopy
}

\author{
S K ROUT*, S PANIGRAHI and J BERA ${ }^{\dagger}$ \\ Department of Physics, 'Department of Ceramic Engineering, National Institute of Technology, Rourkela 769008 , \\ India
}

MS received 31 July 2004; revised 17 February 2005

\begin{abstract}
The ceramics, $\mathrm{SrTiO}_{3}(\mathrm{ST})$ and $0 \cdot 4,0 \cdot 8$ atom\% Ni doped $\mathrm{SrTiO}_{3}$, were prepared by solid state reaction route. The average grain size of undoped and doped samples was measured and found to be 1.2, 1.9 and $3.7 \mu \mathrm{m}$, respectively. The impedance measurements were conducted at $400-600^{\circ} \mathrm{C}$ to separate grain and grain boundary contributions. The grain and grain boundaries relaxation frequencies were shifted to higher frequency with temperature. Bulk resistance of doped and undoped ST ceramics was more or less the same. Single grain boundary resistance of doped sample was higher than that of undoped one, indicating that GB resistance increases with acceptor doping. Activation energies were calculated to confirm the same.
\end{abstract}

Keywords. Ni doped $\mathrm{SrTiO}_{3}$; impedance spectroscopy; grain; grain boundary; acceptor.

\section{Introduction}

In commercial multilayer ceramic capacitors (MLCs), perovskite structure titanate are frequently used as high permittivity dielectrics. Alkaline earth titanates like $\mathrm{BaTiO}_{3}$, $\mathrm{SrTiO}_{3}$ etc are widely used in microelectronic devices due to their excellent dielectric properties and high thermal stability. $\mathrm{SrTiO}_{3}$, a technologically highly important class of perovskite material, is used in PTC resistors, capacitors and sensors (Daniels et al 1978; Gerblinger and Meixher 1991). Important applications of $\mathrm{SrTiO}_{3}$ are as substrates for the heteroepitaxial growth of high $T_{\mathrm{c}}$ superconductors (Shlom et al 1990; Kawai et al 1991), in multi-layer capacitors (Dedyk et al 1998) and DRAM devices (Sakuma et al 1990) etc.

Ceramic oxide-based materials like $\mathrm{SrTiO}_{3}$ contain electro-active intragranular (bulk) and intergranular (grain boundary) regions, whose properties depend on the close control of microstructure-stoichiometric relationships. Grain boundaries play an important role in the electrical properties of a variety of ceramic materials and components. In a broad range of ceramics, this shows ionic conduction, mixed ionic-electronic conduction or electronic conduction, grain boundaries act as barriers for the cross transport of the charge carriers. Often, the barrier character of the grain boundary is especially pronounced in the low temperature regime. In this regime, perovskite type titanates such as $\mathrm{BaTiO}_{3}, \mathrm{SrTiO}_{3}$ etc are employed as high permittivity dielectrics for capacitor applications. At elevated temperatures (i.e. well above the Curie tempera-

*Author for correspondence (skrout@ nitrkl.ac.in) ture), the $\mathrm{BaTiO}_{3}$ based ceramics gave clear evidence for GB layers of high resistance, which is interpreted as a Schottky-type depletion space charge effect. For most of the above applications, the titanates must be net acceptor doped to prevent semiconduction in the ceramics (Hagemann et al 1983/84). The high insulation resistance of MLC components is caused mainly by the fact that grain boundaries (GBs) in the dielectric ceramic act as high resistive barriers for the cross transport of charge carriers. Often, under high temperatures and high voltage fields, these resistive grain boundary barriers are reduced and that give rise to substantially field-enhanced leakage currents through the components. That is the reason why detailed dielectric studies, especially at GB barrier interface are important for the perovskite-type titanates.

Acceptor, e.g. Ni-doped $\mathrm{SrTiO}_{3}$, is a mixed conductor of oxygen vacancies and electron holes. The grain boundary properties of acceptor doped $\mathrm{SrTiO}_{3}$ have already been investigated by impedance spectroscopy on polycrystals and bicrystals (Denk et al 1997), in time dependent voltage stapes measurements (Volmann et al 1997), by simulations (Hagenbeck and Waser 1998), using micro-contact measurements (Rodewald et al 2001) etc.

Acceptor doped $\mathrm{SrTiO}_{3}$ is again selected as our main material because it shows a defect and crystal structure very similar to $\mathrm{BaTiO}_{3}$, which still is the most important ceramic in the ceramic industries. In contrast to $\mathrm{BaTiO}_{3}$, $\mathrm{SrTiO}_{3}$ is not ferroelectric over the complete temperature range of technical interest. For our purpose, this is beneficial since the ferroelectric behaviour superimposes an additional degree of complexity by significantly affecting the polarization and charge transport in the material. Acceptor doped $\mathrm{SrTiO}_{3}$ which is the core of the present pa- 
per aims to describe the effect of acceptor concentration on microstructure and electrical properties of the ceramic at elevated temperatures.

\section{Experimental}

Undoped and nickel-doped (0.4 and 0.8 atom\%) strontium titanate were prepared by solid-state reactions from strontium carbonate (S.D. Fine Chem, Mumbai), titanium dioxide (E. Merck India Ltd.) and nickel nitrate (S.D. Fine Chem, Mumbai). All the powders had 99\% purity. The powders were mixed in agate mortar using isopropyl alcohol up to dryness. Mixed powder was calcined at $1200^{\circ} \mathrm{C}$ for $1 \mathrm{~h}$ and then milled again to destroy agglomerates. The calcined powder was characterized by XRD and showed a perovskite structure without evidence of additional phases. For electrical property measurements, the disks were pressed uniaxially at $200 \mathrm{MPa}$ with $2 \mathrm{wt} \%$ PVA solution added as binder and then they were sintered at $1300^{\circ} \mathrm{C}$ for $4 \mathrm{~h}$. All the samples were of uniform thickness $(\sim 1.68 \mathrm{~mm})$ and diameter $(\sim 10.4 \mathrm{~mm})$. Disk density, estimated approximately from its external dimension using Archimedes's principle, was $~ 99 \%$ of theoretical density. Microstructures were taken by optical microscope (figure 2). Typical average grain sizes were estimated for undoped and doped samples using optical microscope. Silver electrodes were printed on to opposite disk faces and were sintered at $700^{\circ} \mathrm{C}$ for $15 \mathrm{~min}$. Impedance measurements were carried out over a range $10 \mathrm{~Hz}-$ $13 \mathrm{MHz}$ using HP-4192A LF impedance analyser, connected with a $\mathrm{PC}$ in the temperature range, $25-600^{\circ} \mathrm{C}$.

\section{Results and discussion}

Figure 1 shows the XRD patterns of the Ni doped and undoped samples. The figure indicates all the samples are of single phase. The patterns were indexed in cubic structure with $c c p 14$ powder indexing program, check cell. The

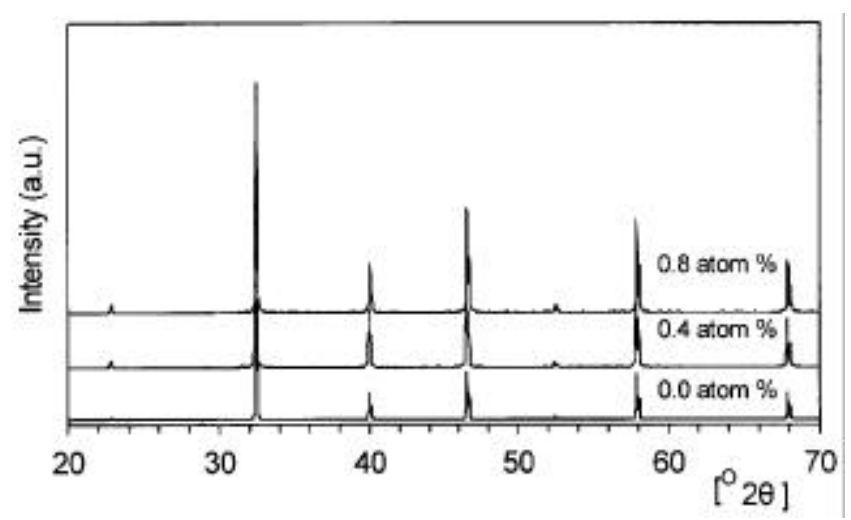

Figure 1. XRD patterns of the undoped, 0.4 atom $\% \mathrm{Ni}$ doped and 0.8 atom\% Ni doped $\mathrm{SrTiO}_{3}$.
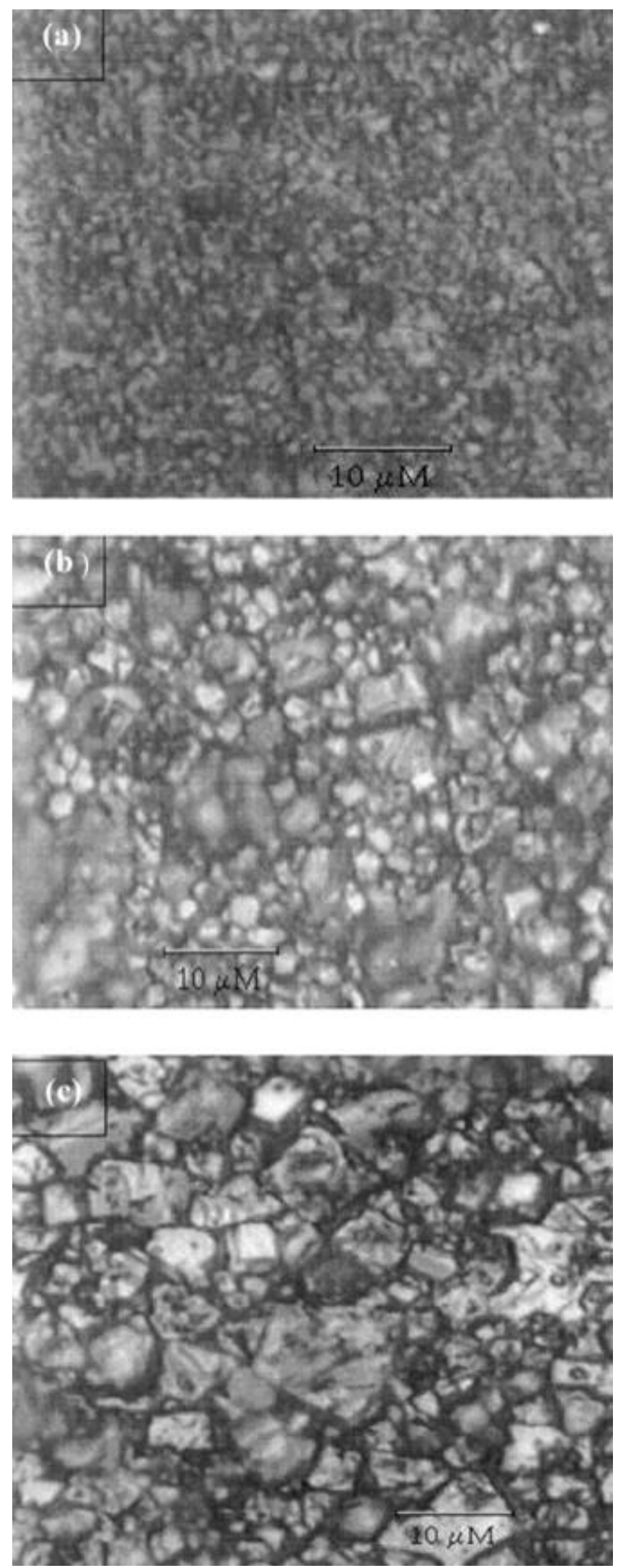

Figure 2. Microstructure of $\mathrm{SrTiO}_{3}$ samples fired at $1300^{\circ} \mathrm{C} / 4 \mathrm{~h}$ : (a) undoped, (b) 0.4 atom\% $\mathrm{Ni}$ doped and (c) 0.8 atom\% $\mathrm{Ni}$ doped. 
lattice parameter was, $a=3 \cdot 8995(2) \AA$, in good agreement with that of JCPDS-Card no. 35-734 ( $a=3.9050 \AA$ ).

Figure 2 shows the optical micrograph of $\mathrm{SrTiO}_{3}$ samples. The average grain sizes are $1.2,1.9$ and $3.7 \mu \mathrm{m}$, for undoped, 0.4 atom $\%$ and 0.8 atom $\%$ Ni-doped samples, respectively. The samples were fired in same firing schedule. Then the differences in grain size may be attributed to the effect of $\mathrm{Ni}$-doping. $\mathrm{NiO}$ may enhance grain growth at the experimental sintering temperature. This may be caused due to the nucleating behaviour of $\mathrm{Ni}$ in the $\mathrm{SrTiO}_{3}$ ceramic.

Grain boundaries act as barriers for the cross transport of the charge carriers. Often, under high temperature and high voltage fields, this grain boundary barriers are reduced, which gives rise to a substantially field enhanced
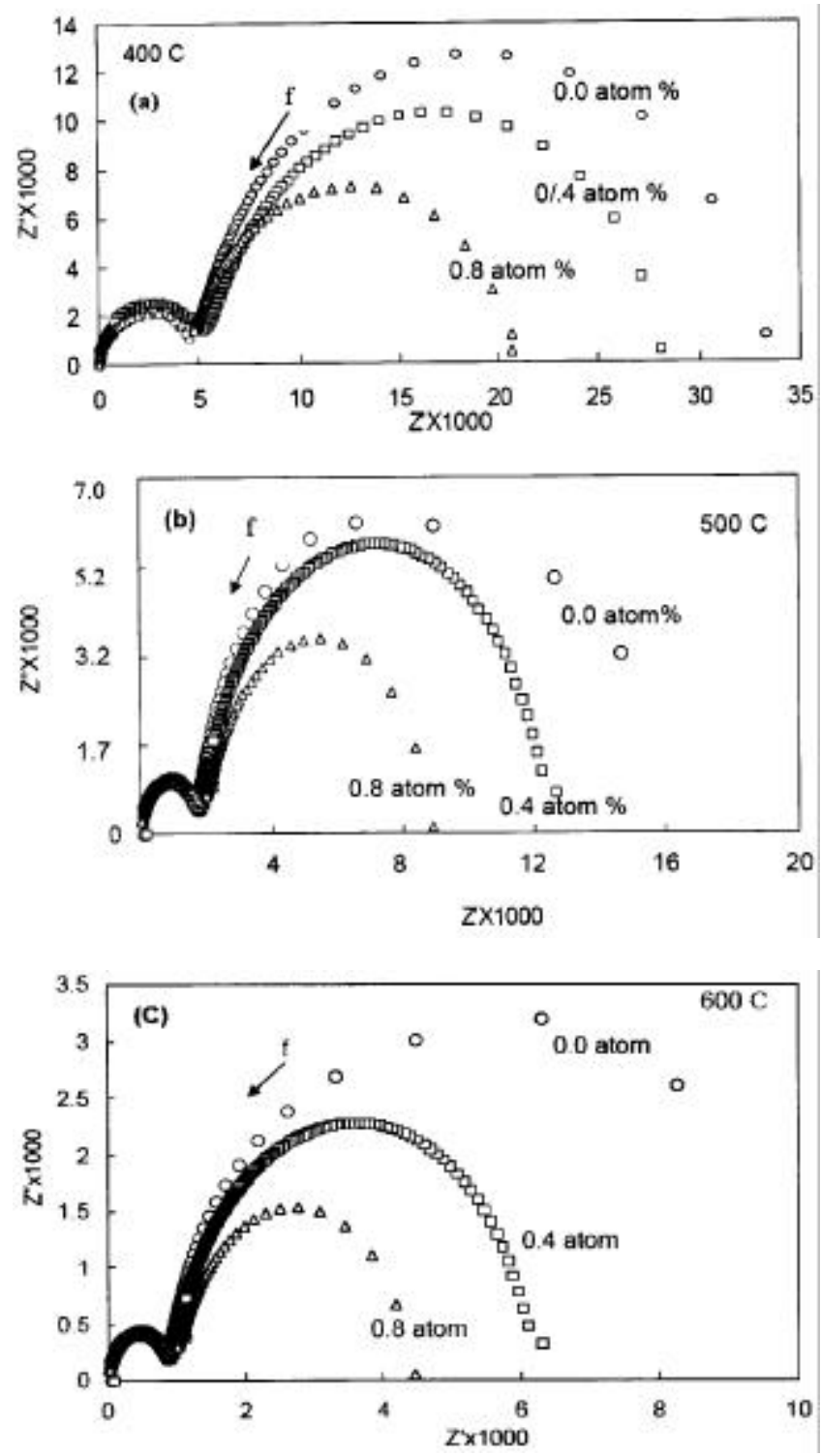

Figure 3. Impedance spectra of the samples at different temperatures $((\mathrm{O}) 0.0$ atom $\%,(\square) 0.4$ atom\% and $(\Delta) 0.8$ atom\% $\mathrm{Ni}$-doped). leakage currents through the components. So a detailed dielectric study, especially at grain boundary barriers, is important for the perovskite type titanates. It is well known that acceptor doped $\mathrm{SrTiO}_{3}$ has positively charged GB interface, which gives rise to an electrostatic repulsion of the positively charged mobile oxygen vacancy at both sides of the boundary.

It is known that overall grain boundary resistance, $R_{\mathrm{gb}}$, increases with the decrease in grain size due to the increase in number of boundaries per unit thickness. A typical grain-boundary thickness of nickel-doped materials was estimated to be of the order of $100 \mathrm{~nm}$ (Volmann et al 1997), which is usually much lower than the average grain size. Hence, no significant changes are expected in bulk resistance on changing the grain size.

Figure 3 shows the impedance spectra of the samples at three representative temperatures. The amplitude of high frequency arc of the impedance spectra for three samples having different grain sizes does not vary significantly with increasing grain size (table 1) and is ascribed to the bulk resistance $\left(R_{\mathrm{b}}\right)$. Low frequency arc was not found at $<400^{\circ} \mathrm{C}$ for the three samples, which may be due to the effect of electrode relaxation process overlapping with the grain boundary relaxation process. However, with increase in temperature $\left(>400^{\circ} \mathrm{C}\right)$ the low frequency arc was found as shown in figure 3 . The low frequency arc is ascribed to $R_{\mathrm{gb}}$ because arc amplitude decreases with increase in temperature.

Figure 3 shows two semicircles (arc) for all samples representing two $\mathrm{RC}$ elements. The high frequency arc corresponds to $R_{\mathrm{b}} C_{\mathrm{b}}$ response and low frequency arc corresponds to $R_{\mathrm{gb}} C_{\mathrm{gb}}$ response. The spectrum clearly indicates a combination of bulk and grain boundary impedances connected in series. The impedance for this circuit, $Z^{*}=$ $\left[R_{\mathrm{b}}^{-1}+j \omega C_{\mathrm{b}}\right]^{-1}+\left[R_{\mathrm{gb}}^{-1}+j \omega C_{\mathrm{gb}}\right]^{-1}$, where the subscripts $\mathrm{b}$ and gb refer to bulk and grain boundary. Grain and grain boundary relaxation frequencies for three different samples are tabulated in table 1 . Both the grain and grain boundary relaxation frequencies increase with increase in dopant concentration, which may be due to increase in average grain size. It is well known that $R_{\mathrm{gb}}$ increases with decrease in grain size due to the increase in number of boundaries per unit thickness (Jurado et al 2000). As in the case of samples of unequal grain thickness, to compare the actual grain boundary resistivity, grain size contribution is considered by the relation,

$$
\rho_{\mathrm{gb}}=d_{\mathrm{g}} A R_{\mathrm{gb}} / d_{\mathrm{gb}} L,
$$

where $d_{\mathrm{gb}}$ is the thickness of the grain boundary layer, $d_{\mathrm{g}}$ the average grain size, $L$ the sample thickness, $A$ the electrode area and $R_{\mathrm{gb}}$ the grain boundary resistance. Volmann and Waser (1994) studied a typical grain boundary thickness of $\mathrm{Ni}$ doped materials which was estimated to be of the order of $100 \mathrm{~nm}$, which is usually much less than the average grain size. $R_{\mathrm{b}}$ of all three samples are more or less same at these representative temperatures 
Table 1. Fitting parameter and activation energy obtained from impedance spectra (figure 3).

\begin{tabular}{|c|c|c|c|c|c|}
\hline Sample (Av. grain size) & Parameter & $400^{\circ} \mathrm{C}$ & $500^{\circ} \mathrm{C}$ & $600^{\circ} \mathrm{C}$ & Activation energy $(\mathrm{eV})$ \\
\hline \multirow[t]{4}{*}{ Undoped $(1 \cdot 2 \mu \mathrm{m})$} & $R_{\mathrm{b}}(\mathrm{k} \Omega)$ & 5 & 2 & 1 & 0.96 (B) \\
\hline & $f_{\max }(\mathrm{bulk}) / \mathrm{kHz}$ & $27 \cdot 8$ & 310 & 1550 & 0.98 (B) \\
\hline & $R_{\mathrm{gb}}(\mathrm{k} \Omega)$ & 29 & 16 & 9 & $1 \cdot 51(\mathrm{~GB})$ \\
\hline & $f_{\max }(\mathrm{GB}) / \mathrm{kHz}$ & $0 \cdot 12$ & $4 \cdot 8$ & $39 \cdot 8$ & $1.48(\mathrm{~GB})$ \\
\hline \multirow[t]{4}{*}{0.4 atom $\%$ Ni doped $(1.9 \mu \mathrm{m})$} & $R_{\mathrm{b}}(\mathrm{k} \Omega)$ & 5 & 2 & 1 & $0.97(\mathrm{~B})$ \\
\hline & $f_{\max }($ bulk $) / \mathrm{kHz}$ & 40 & 400 & 2400 & $0.99(\mathrm{~B})$ \\
\hline & $R_{\mathrm{gb}}(\mathrm{k} \Omega)$ & 23 & 10 & $5 \cdot 2$ & $1 \cdot 5(\mathrm{~GB})$ \\
\hline & $f_{\max }(\mathrm{GB}) / \mathrm{kHz}$ & $0 \cdot 31$ & $6 \cdot 8$ & $90 \cdot 01$ & $1.42(\mathrm{~GB})$ \\
\hline \multirow[t]{4}{*}{0.8 atom $\%$ Ni doped $(3.7 \mu \mathrm{m})$} & $R_{\mathrm{b}}(\mathrm{k} \Omega)$ & 5 & 2 & 1 & $0.98(\mathrm{~B})$ \\
\hline & $f_{\max }($ bulk $) / \mathrm{kHz}$ & 45 & 470 & 2700 & $1.01(\mathrm{~B})$ \\
\hline & $R_{\mathrm{gb}}(\mathrm{k} \Omega)$ & 16 & 7 & $3 \cdot 5$ & $1 \cdot 51(\mathrm{~GB})$ \\
\hline & $f_{\max }(\mathrm{GB}) / \mathrm{kHz}$ & $0 \cdot 53$ & $9 \cdot 7$ & $230 \cdot 01$ & $1.52(\mathrm{~GB})$ \\
\hline
\end{tabular}
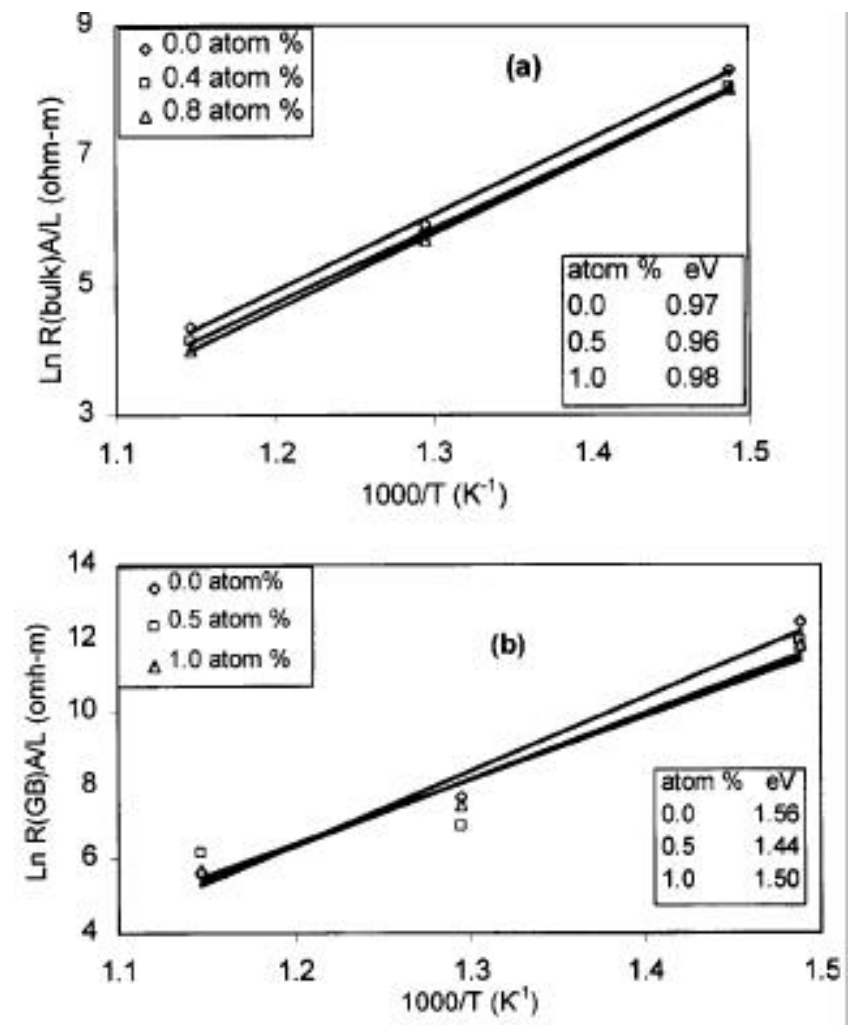

Figures 4(a)-(b). Temperature dependence of bulk and grain boundary resistivity obtained from impedance data.

due to same type of bulk grain behaviour. Hence, no significant changes are expected in the bulk resistance on changing the average grain size. In our case $A$ and $L$ are same for different samples. $d_{\mathrm{gb}}$ is $100 \mathrm{~nm}$ for $\mathrm{Ni}$ doped materials, as suggested by Volmann and Waser (1994). So to compare the resistivity, only average grain size should be multiplied with the observed resistance value (table 1) from the impedance spectra, i.e. the relation $\left(\rho_{\mathrm{gb}}=d_{\mathrm{g}} A R_{\mathrm{gb}} / d_{\mathrm{gb}} L\right)$ can be considered as grain size $\times R_{\mathrm{gb}}$.
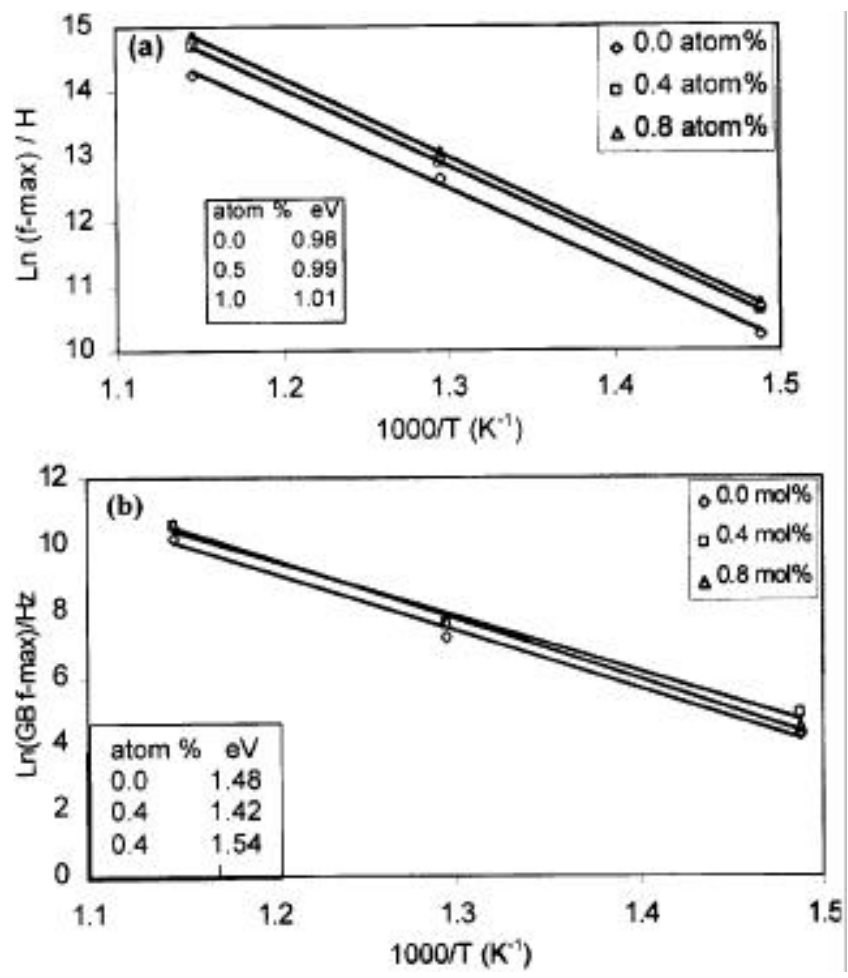

Figures 5(a)-(b). Temperature dependence of bulk and grain boundary resistivity obtained from respective relaxation frequencies.

Hence, using this relation it could be clearly observed that $\rho_{\mathrm{gb}}$ (undoped) $<\rho_{\mathrm{gb}}(0.4$ atom $\%$ doped $)<\rho_{\mathrm{gb}}(0.8$ atom $\%$ doped). This is due to the formation of highly resistive grain boundary due to $\mathrm{Ni}$ doping. Again it can be clearly seen that $\rho_{\mathrm{gb}}\left(600^{\circ} \mathrm{C}\right)<\rho_{\mathrm{gb}}\left(500^{\circ} \mathrm{C}\right)<\rho_{\mathrm{gb}}\left(400^{\circ} \mathrm{C}\right)$ for all samples due to the increase in leakage current at the grain boundary.

Figures 4(a) and (b) show the temperature dependence of bulk resistivity and grain boundary resistivity, respec- 
tively obtained from the impedance data. Bulk resistance follows generic Arrhenius law, giving an activation energy of about $\sim 1 \mathrm{eV}$ (figure 4(a)), which is close to the value reported by Volmann et al (1997). Again grain boundary activation energy is calculated and observed to be about $1.5 \mathrm{eV}$ for 0.8 atom $\%$ doped sample and $1.56 \mathrm{eV}$ for undoped sample which are slightly lower than the $w$ type conductivity profile $(\sim 1.6 \mathrm{eV})$ reported for polycrystalline Ni doped samples (Volmann et al 1997), but for activation energy of 0.4 atom $\%$ doped sample, it was $1.44 \mathrm{eV}$ which is lower than the $w$ type conductivity and this may be due to very small and non-uniform grain size.

Figures 5(a) and (b) show the temperature dependence of bulk resistivity and grain boundary resistivity, respectively obtained from the relaxation frequency. Here the bulk resistance follows the generic Arrhenius law giving activation energy very close to $1 \mathrm{eV}$ as reported earlier (Volmann et al 1997). Again grain boundary activation energy obtained from figure 5(b) more or less agreed with the values that were calculated from resistance data (figure 4(b)).

\section{Conclusions}

Ni doped sample showed higher resistivity than undoped sample and that may be explained by the formation of thicker space charge layer at the interface due to higher concentration of acceptor doping. Fine-grained ST ceramics are desirable for higher GB resistivity.

\section{References}

Daniels J, Hardl K H and Wernicke R 1978 Philips Tech. Rev. 3873

Denk J, Claus J and Maier J 1997 J. Electrochem. Soc. 1443526

Dedyk A I, Karmanenko S F, Leppavuori S and Sakharov V I 1998 J. Phys. France 8217

Gerblinger J and Meixner H 1991 Sens. Actuators B4 99

Hagemann H J, Hennings D and Wernicke R 1983/84 Philips Tech. Rev. 4189

Hagenbeck R and Waser R 1998 J. Appl. Phys. 832083

Jurado R J, Colomer M T and Frade J R 2000 J. Am. Ceram. Soc. 832715

Kawai M, Watanabe S and Hanada T 1991 J. Cryst. Growth 112 745

Park M B and Cho N H 2001 J. Am. Ceram. Soc. 841917

Rodewald S, Fleig J and Maier J 2001 J. Am. Ceram. Soc. 84 521

Sakuma T, Yamamichi S, Matsubara S, Yamaguchi $\mathrm{H}$ and Miyasaka Y 1990 Appl. Phys. Lett. 572431

Shlom D G, Marshall A F, Sizemore Z J, Chen J N, Bozovic I, von Dessonneck K E, Harris J S and Bravman J C $1990 \mathrm{~J}$. Cryst. Growth 102361

Volmann M and Waser R 1994 J. Am. Ceram. Soc. 77235

Volmann M, Hagenbeck R and Waser R 1997 J. Am. Ceram. Soc. 802301 